NASA Technical Memorandum 107003

\title{
Parametrics on 2D Navier-Stokes Analysis of a Mach 2.68 Bifurcated Rectangular Mixed-Compression Inlet
}

M. Mizukami and J.D. Saunders

Lewis Research Center

Cleveland, Ohio

Prepared for the

31st Joint Propulsion Conference and Exhibit cosponsored by AIAA, ASME, SAE, and ASEE San Diego, California, July 10-12, 1995

National Aeronautics and

Space Administration 


\title{
PARAMETRICS ON 2D NAVIER-STOKES ANALYSIS OF A MACH 2.68 BIFURCATED RECTANGULAR MIXED-COMPRESSION INLET
}

\author{
M. Mizukami and J. D. Saunders \\ NASA Lewis Research Center \\ Cleveland, Ohio 44135
}

$\underline{\text { Abstract }}$

The supersonic diffuser of a Mach 2.68 bifurcated, rectangular, mixed-compression inlet was analyzed using a two-dimensional (2D) Navier-Stokes flow solver. Parametric studies were performed on turbulence models, computational grids and bleed models. The computed flowfield was substantially different from the original inviscid design, due to interactions of shocks, boundary layers, and bleed. Good agreement with experimental data was obtained in many aspects. Many of the discrepancies were thought to originate primarily from 3D effects. Therefore, a balance should be struck between expending resources on a high fidelity $2 \mathrm{D}$ simulation, and the inherent limitations of $2 \mathrm{D}$ analysis. The solutions were fairly insensitive to turbulence models, grids and bleed models. Overall, the $k-\varepsilon$ turbulence model, and the bleed models based on unchoked bleed hole discharge coefficients or uniform velocity are recommended. 2D Navier-Stokes methods appear to be a useful tool for the design and analysis of supersonic inlets, by providing a higher fidelity simulation of the inlet flowfield than inviscid methods, in a reasonable turnaround time.

\begin{tabular}{ll} 
& \multicolumn{1}{c}{ Nomenclature } \\
$x$ & axial coordinate, from ramp tip \\
$y$ & vertical coordinate, from centerline \\
$h_{c}$ & cowl half-height \\
$p$ & static pressure \\
$p T$ & total pressure \\
$\rho$ & density \\
$u$ & velocity
\end{tabular}

\begin{tabular}{ll}
\multicolumn{2}{l}{ Subscripts } \\
$o$ & freestream \\
$w$ & wall
\end{tabular}

Copyright (C) 1995 by the American Institute of Aeronautics and Astronautics, Inc. No copyright is asserted in the United States under Title 17, U.S. Code. The U.S. government has a royalty-free license to exercise all rights under the copyright claimed herein for Governmental Purposes. All other rights are reserved by the copyright owner.
Introduction

The inlet is an essential part of the propulsion system on a supersonic cruise aircraft. ${ }^{1,2}$ A supersonic inlet consists of two portions: the supersonic diffuser is the forward part where shock waves are used to compress the flow, and the subsonic diffuser is the aft part. To achieve high total pressure recovery and low cowl drag at cruise Mach number above 2.0 to 2.2 , a mixed-compression scheme is necessary, where the supersonic compression is split between an 'external' part forward of the cowl lip and an 'internal' part aft.

For good recovery while retaining a margin of stability, mixed-compression inlets are designed to compress the freestream flow to about Mach 1.3 at the exit of the supersonic diffuser or 'throat'. When the normal or 'terminal' shock is positioned right at the throat, the inlet is operating at the design or 'critical' point. If the normal shock moves downstream of the throat, the shock loss increases; this range of operation is termed 'supercritical'.

Due to the sensitivity of the nearly sonic flow in the throat region, even a slight perturbation caused by atmospheric gusts, engine airflow transients, or shockboundary layer interactions can radically alter the inlet flow. The result can be an 'unstart' or 'buzz' condition, where the normal shock has been expelled forward in front of the inlet, and the flow becomes highly unsteady. Inlet total pressure recovery is drastically reduced, and severe forces may result on the aircraft.

To help prevent unstarts, a fraction of the airflow is removed through small openings in the inlet walls in a process known as 'bleed'. Bleed helps to stabilize the normal shock, improve the shock-boundary layer interactions, and increase the local Mach number to prevent subsonic regions ${ }^{3}$. Also, bleed helps to keep the flow well conditioned at on-design operation. Due to the complexity of the flow, design of bleed is largely based on empirical guidelines.

In a wind tunnel test, to simulate external disturbances that may cause unstarts, the angle of attack and freestream Mach number are varied. The angle of attack or Mach number reduction the inlet can tolerate without unstarting is a measure of the inlet's 'operability limit'.

Therefore, the issues with a mixed compression scheme include the possibility of inlet unstarts, the need 
for boundary layer control and normal shock stability systems such as bleed, and increased mechanical complexity. ${ }^{4}$ The design is a balance between the conflicting requirements of internal performance, external drag, weight and operability.

A medium scale model of the Mach 2.68 bifurcated rectangular mixed compression inlet with $30 \%$ internal area contraction was tested in the NASA Lewis $10 \times 10$ foot cross section supersonic wind tunnel (Figs. 1 and 2). ${ }^{5}$ The freestream Reynolds number at Mach 2.68 was $2.5 \times 10^{6}$ per foot, and the cowl half-height $\left(h_{c}\right)$ was 10.67 inches. Figure 3 shows the theoretical shock structure designed by using the method of characteristics. The initial ramp shock is followed by an isentropic compression fan, both focused on the cowl lip. The initial cowl shock is also followed by an isentropic compression fan. The cowl shock is canceled at the ramp shoulder, and the compression fan is canceled on the curved ramp surface. In the test program, parametrics were performed on the freestream Mach number, angle of attack, bleed patterns, subsonic diffuser vortex generator patterns, and ramp positions. Three configurations were selected for detailed study, two of which had the ability to self-start following an unstart without active controls. One of the self starting configurations gave 89 percent total pressure recovery and 16 percent distortion at the compressor face station, with a bleed massflow of 6.9 percent of the capture massflow. A steeper than expected ramp tip shock resulted in a high spillage of 4 to 5 percent of capture.

Supersonic diffuser geometries for mixed compression inlets are generally designed using inviscid analyses. The method of characteristics is typically used, because it produces exact inviscid results, and because the very fast turnaround time is amenable to design. Euler finite difference analysis has been shown to produce nearly the same results as the method of characteristics, and it is more easily extended to a three dimensional (3D) computation ${ }^{6}$. However, Euler analysis is not exact due to truncation errors, and it requires more computational resources than the method of characteristics. The actual flowfield in the inlet differs appreciably from the inviscid analysis, due to viscous effects and viscous-inviscid interactions, such as boundary layer buildup and shock-boundary layer interactions. Therefore, a number of analysis techniques incorporating these effects have been developed, to more accurately simulate the flowfield.

In a zonal approach, the flowfield is partitioned into distinct areas depending on the dominant physics, and each area is analyzed using a separate technique. Typically, the inviscid core is computed using the method of characteristics, near wall areas are solved with a boundary layer code, bleed regions are modeled by manipulating the boundary layer profiles, and oblique shock-boundary layer interactions are solved using control volume analysis. The zonal approach is useful for rapidly evaluating a large number of configurations, and for determining boundary layer properties in order to place bleed regions. However, more complex flows, such as corner flows, subsonic regions, separations and vortices, are not properly modeled. Therefore, 3D flows, terminal shocks, and operability limits are not accurately simulated. Using the zonal approach, Vadyak et al. ${ }^{7}$ obtained remarkably good agreement with data on a 3D analysis of an axisymmetric inlet at incidence. Reyhner and Hickcox ${ }^{8}$ coupled the inviscid core flow with the boundary layer, by moving the wall by a distance equal to the displacement thickness, and recomputing the inviscid core flow; they obtained good agreement with data for an axisymmetric inlet, except near the normal shock and where subsonic flow was predicted.

In reduced or 'parabolized' Navier Stokes (PNS) analysis, the streamwise diffusion term is neglected. For supersonic flow, this allows the solution to be marched downstream in space, computing only one axial plane at a time. Therefore, PNS is computationally efficient, and it is well suited for modeling supersonic diffusers, including 3D phenomena. However, PNS may not properly simulate inherently elliptical flow features such as large subsonic regions and separated flows, so it is not suitable for determining operability limits. Anderson and Towne obtained good agreement with data using this method. Buggeln et al. ${ }^{10}$ obtained reasonable agreement with limited data for a 3D analysis of a rectangular inlet.

Although full Navier-Stokes analysis is computationally expensive, its main advantage is generality. Complex flows and interactions such as corner flows, glancing shock-boundary layer interactions and secondary flows can be modeled, at least in principle. Therefore, within the limits of available computational resources, almost all aspects of the inlet flow could be modeled, including 3D effects, operability, terminal shock, and subsonic diffuser flow. General purpose Navier-Stokes solvers can be used with only minor modifications, minimizing the required code development and validation effort. However, many issues remain to be considered, such as truncation error, accuracy of turbulence models in complex flows, and grid sensitivity ${ }^{11}$. A number of Navier-Stokes solutions of mixed compression inlets have been reported. Knight ${ }^{12}$ demonstrated a case where Navier Stokes analysis produced more accurate results than the zonal method. Chyu et al $(1986)^{13}$ produced axisymmetric solutions for supercritical, critical and subcritical operations, but surprisingly with no bleed 
regions. Shigematsu et al ${ }^{14}$ reported a two-dimensional (2D) solution of a rectangular inlet in supercritical operation, and a 3D solution of the supersonic diffuser, both using the Baldwin-Lomax algebraic turbulence model; they obtained qualitative agreement with data in the limited comparisons, and stated that the turbulence model would have to be investigated to improve the results. Saunders and Keith ${ }^{15}$ studied an axisymmetric inlet including the terminal shock and the subsonic diffuser; turbulence modeling, bleed modeling and terminal shock oscillations were found to be issues in this type of calculation. Reddy and Weir ${ }^{16}$ showed substantial 3D effects in a Mach 5 rectangular inlet, and obtained good qualitative agreement with data, using an algebraic turbulence model and a simple uniform mass flux bleed boundary condition; it was suggested that a better turbulence model would improve simulation of the corner flows. Fujimoto et al ${ }^{17}$ and Omi et $\mathrm{al}^{18}$ simulated the inlet under supercritical, critical and subcritical conditions in a 2D calculation, but with no experimental comparisons. Fujimoto and Niwa ${ }^{19}$ reported a 3D calculation of a rectangular inlet including the terminal shock; good agreement was shown with data, and a vortex was seen along the cowl shocksidewall interaction. Chung $^{20}$ investigated a new engine face boundary condition and the behavior of the terminal shock for an axisymmetric inlet, mainly using Euler analysis. Grasso and Marini ${ }^{21}$ made a 2D calculation including the terminal shock, and obtained good agreement in limited comparisons with data. Chyu et al (1992) ${ }^{22}$ investigated several boundary conditions for porous bleed, including their ability to stabilize the terminal shock; some of the bleed boundary conditions required extensive prior knowledge of the bleed region flowfield. Mayer and Paynter ${ }^{23}$ investigated engine face and bleed boundary conditions for an axisymmetric inlet including the terminal shock, using Euler analysis. Freskos and Penanhoat ${ }^{24}$ produced 2D and 3D solutions of a Mach 1.865 rectangular inlet including the terminal shock, but did not report any comparisons with data.

In the present study, the supersonic diffuser of a Mach 2.68 bifurcated, rectangular, mixed-compression inlet was analyzed using a 2D Navier-Stokes flow solver. The objectives were three-fold. First, the accuracy and limitations of $2 \mathrm{D}$ Navier-Stokes analysis on a rectangular inlet were explored, relative to the original method of characteristics design, and to the experimental data from wind tunnel testing. Second, parametric studies were performed on turbulence models, solution grids and bleed models. Third, this serves as a validation case for this flow solver on supersonic internal flows with porous bleed and adverse pressure gradients.

\section{Methods}

2D Navier-Stokes analysis was performed on the supersonic diffuser, at the design Mach number and ramp positions, with the inlet operating at a supercritical condition. Taking advantage of symmetry, only one half of the bifurcated inlet was analyzed. Table I summarizes the configurations and results. Two bleed configurations were investigated. The configuration called 'SS1' has higher bleed mass flow rates, and was experimentally shown to be self-starting. The 'NSS' configuration has lower bleed, but is not self-starting. Experimental bleed patterns and bleed flow rates were adapted to equivalent $2 \mathrm{D}$ cases, by neglecting the sidewall and corner bleed regions, and by reducing the bleed mass flows by the estimated massflow through the sidewall and corner bleeds.

The flow solver used in the present study is NPARC, a Navier-Stokes solver used extensively by government and industry to analyze compressible flows. ${ }^{25.26}$ NPARC was derived from PARC, which in turn was derived from the ARC code. It is under continuing development and is supported by an alliance of NASA Lewis Research Center (LeRC) and the USAF Arnold Engineering and Development Center (AEDC).

The governing equations in the flow solver are the time-dependent, Reynolds-averaged Navier-Stokes equations with a perfect gas relationship and Fourier's heat conduction law. These equations are discretized in conservation law form with respect to general curvilinear coordinates, and solved with the Beam and Warming approximate factorization algorithm. Although the time dependent formulation of the governing equations are used, the code is intended for steady state simulations, because not all portions of the present version of the code are time-accurate.

Grid sensitivity was evaluated by running three different grids. All three were generated using GENIE3D, a graphical version of the 3D INGRID grid generation program. $^{27}$ In an effort to resolve the shock waves more crisply, the grid was adapted as much as possible to the initial ramp shock and the cowl shock by slanting the cross-stream grid lines, while limiting the maximum grid skewness to about $45^{\circ}$ (Fig. 4). The cowl lip was as sharp as the grid packing at the wall would allow. The first grid generated was the 'coarse' grid, with dimensions of $180 \times 140$, and a typical y+ value of the first grid point off the wall of about 6 . In order to determine grid sensitivity, the 'fine' grid was generated, by doubling the resolution of the coarse grid in both directions; its dimensions are $360 \times 280$ with a typical $y+$ value of 3 . Based on preliminary results using the coarse and fine grids, the 'improved' grid was generated, with the grid at the wall packed to $y+$ of less 
than 5 as required by the turbulence models, and with an overall grid size small enough such that converged results can be obtained overnight on a midrange workstation. The improved grid has dimensions of 210 $x 140$, a typical $y+$ value of 2 , and grid resolution in the inviscid core region intermediate to that of the coarse and fine grids. For this grid sensitivity study, the $\mathrm{k}-\varepsilon$ turbulence model, uniform velocity bleed model and the NSS configuration were used. The uniform velocity bleed model is robust because it imposes a constant mass flow regardless of the inlet flow conditions, and it is thought to provide a more realistic mass flux distribution than the uniform mass flux bleed model. The NSS configuration, which has smaller bleed regions and lower bleed mass flows, was used in order to minimize the impact of the mass flux distribution resulting from the simplistic bleed model.

Three turbulence models were examined. The first is the Chien $\mathrm{k}-\varepsilon$ two-equation model ${ }^{28}$, including the compressibility modifications of Nichols ${ }^{29} . \mathrm{k}-\varepsilon$ and other two equation models are generally applicable to a wide range of flows, and have reasonable computational requirements compared to more elaborate techniques, such as Reynolds stress modeling or large eddy simulation. Therefore, two-equation models are often preferred for engineering applications. The second is the Baldwin-Barth one equation model ${ }^{30}$. The third and least computationally intensive is the Baldwin-Lomax algebraic model ${ }^{31}$, which is applicable to attached or mildly separated wall bounded flows. All walls were assumed to be turbulent. For this turbulence model parametric study, the improved grid, the NSS configuration and the uniform velocity bleed model were used.

Four models for porous bleed were evaluated. In all four, the tangential velocity at the wall was zero as per the no slip condition, the static pressure and temperature gradients normal to the wall were prescribed to be zero, and no attempt was made to model the 'roughness' effect of the porous bleed surface. ${ }^{32}$ The four models differed in how the bleed flow velocity normal to the wall was determined. The 'uniform velocity' model assigns a uniform velocity normal to the wall over the bleed region, based on the desired mass flow rate. The 'uniform mass flux' model assigns a uniform mass flux over the bleed region, based on the desired mass flow rate. The 'choked hole' model assigns local wall normal velocities based on the desired mass flow rate and experimentally determined discharge coefficients of choked bleed holes $^{33}$. Because the bleed holes in the experiment were not choked, the numerical porosity of the bleed region was adjusted until the desired mass flow was obtained, giving an equivalent 'choked' porosity. The 'unchoked hole' model determines local wall normal velocities based on discharge coefficients of unchoked bleed holes, bleed region porosity, and plenum back pressure. Note that no prior knowledge of the bleed mass flow rate is required, and in fact, the mass flow will vary depending on the inlet flow conditions. For this bleed model parametric study, the SS1 configuration, $k-\varepsilon$ turbulence model and the uniform velocity bleed model were used. The SS1 configuration was used because it has extended bleed regions and higher bleed mass flow rates, which highlights the differences between the bleed models.

\section{$\underline{\text { Results and Discussion }}$}

Results for the coarse, fine and improved grids were similar. Even the boundary layer profiles, measured just upstream of (ramp BL rake 1) and downstream of (ramp BL rake 2) the ramp shoulder, were similar for all three grids (Fig. 5). The fine grid has about four times more grid points, and requires more than four times the computer resources to obtain a solution than the other two grids. The improved grid appears to be the best compromise, having reasonable computational requirements as well as better packing at the wall than the coarse grid, although it does not resolve the internal waves as crisply as the fine grid.

All three turbulence models produced fairly similar results (Fig. 6), which is not surprising considering that the flow is attached and well behaved in general. However, the Baldwin-Barth model produced somewhat strange boundary layer profiles. The $k-\varepsilon$ model is recommended, despite its greater computational requirements, because it is applicable to a wide range of flows, and because it is expected to more accurately simulate 3D flows.

Results using the four different porous bleed models were surprisingly similar (Fig. 7), even though the bleed region mass flux distributions were substantially different. It can be seen that both the choked hole and unchoked hole models vary the mass flux depending on local conditions. Also, the uniform velocity model better approximates the mass flux distribution of the unchoked hole model than the uniform mass flux model. If the bleed hole geometry, porosity, and the plenum backpressure (if unchoked) are known, then the unchoked hole model is the most sophisticated simulation, and it requires no prior knowledge of the bleed mass flow rates. If the flow rates are known, but the details of the bleed configurations are unknown, then the uniform velocity model appears to produce a good solution. However, the uniform velocity model is not suitable for investigating stability or operability limits, where bleed 
mass flow rates must vary in response to the off-design operating conditions, to help stabilize the flow.

Figures 8 and 9 show computed results for the NSS and SS1 configurations respectively, using the improved grid, $\mathrm{k}-\varepsilon$ turbulence model, and the unchoked bleed model. Mach contours with bleed mass flux vectors, ramp pressures, cowl pressures, and ramp boundary layer rake profiles are plotted; comparisons are made with experimental data and inviscid theory. Table I shows the mass flow rates, cowl shock impingement points and recoveries.

As seen in the Mach contour plots and surface pressure plots, there are substantial waves in the internal flow. These waves are not predicted by inviscid theory.

Present results and experiment both show steeper shocks and increased compression than theory, as a result of boundary layer displacement effects. The cowl shock impinges on the ramp surface in front of the shoulder and reflects off, instead of being canceled; the impingement point is well predicted.

Bleed regions are seen to generate waves, most likely due to flow turning. At the start of the bleed region, the streamlines turn toward the wall, producing an expansion wave. Over the bleed region, the streamlines angle into the wall. At the end of the bleed region, the streamlines turn sharply away from the wall as the boundary layer thickens rapidly at the start of the solid wall, producing a compression wave.

The experiment indicates an unusually high spillage flow rate, most likely due to boundary layer displacement steepening the ramp shock, and the resulting spillage over the sidewalls. The present $2 \mathrm{D}$ analysis does not account for spillage over the sidewalls, and therefore predicts a lower spillage.

Ramp and cowl pressures for the NSS configuration show reasonably good agreement between present results and experiment. For the cowl pressures in the NSS configuration, the high and low pressure spikes near the throat are well predicted. However, cowl pressures for the SS1 configuration show a substantial discrepancy in the throat region. No apparent physical origin can be identified in a 2D flowfield for the high pressure measured experimentally near $x / h_{c}=3.1$ and the lower pressure around $x / h_{C}=3.5$. It is difficult to attribute such a large discrepancy to numerical or modeling error in a relatively well-behaved flow such as this. Therefore, it is speculated that the differences may be $3 \mathrm{D}$ in origin. The glancing interaction of the cowl shock and the sidewall boundary layer may be adverse enough to produce a shock wave off the sidewall, which propagates diagonally downstream, and appears as a high pressure at the cowl centerline around $\mathrm{x} / \mathrm{h}_{\mathrm{c}}=3.1$. The expansion wave from the forward sidewall bleed may also propagate and appear as a lower pressure around $x / h_{c}=3.5$. Supporting this speculation are the position of the forward sidewall bleed relative to the measured expansion region, the position of the cowl leading edge relative to the measured high pressure region, and the fact that the higher bleed SS1 configuration shows a larger discrepancy. Furthermore, waves arising from the two sidewalls would constructively interfere at the centerline where the surface pressure measurements were taken, producing a greater pressure change.

The boundary layer thickness upstream of the bleed regions, at ramp BL rake 1, is underpredicted. However, the fact that all three turbulence models underpredict the thickness by almost exactly the same amount suggests that the discrepancy might not be due to modeling error. Instead, the thicker boundary layer may be due to other factors, such as steps in the model ramp surface, or 3D compression waves from the sidewalls. Profile agreement downstream of the ramp shoulder bleed, at ramp BL rake 2, is fair but inconclusive, because the upstream profiles at ramp BL rake 1 do not match well.

2D Navier-Stokes analysis appears to be a useful tool for the design and analysis of supersonic inlets. After the supersonic diffuser geometry is initially laid out using the method of characteristics, Navier-Stokes analysis can provide a higher fidelity solution by taking into account viscosity and bleed. Turnaround time is still fast enough to be useful in the design process. However, many of the observed discrepancies appear to be 3D in origin, indicating that a balance should be struck between expending resources on a very fine mesh, high fidelity 2D simulation, and the inherent limitations of 2D analysis.

Based on lessons learned from the present parametric study, 3D analysis of the inlet is presently underway. The objectives are to further validate the code and to gain additional fluid dynamic insight, by accounting for 3D effects such as sidewall bleed, spillage over the sidewalls, and glancing cowl shock sidewall boundary layer interactions.

\section{Conclusions}

The supersonic diffuser of a Mach 2.68 bifurcated, rectangular, mixed-compression inlet was analyzed using a 2D Navier-Stokes flow solver. Parametric studies were performed on turbulence models, computational grids and bleed models. Comparisons were made with experimental data and with the original inviscid design. The major conclusions are as follows.

1. The computed flowfield was substantially 
different from the original inviscid design, due to interactions of shocks, boundary layers, and bleed. Good agreement with experimental data was obtained in many aspects.

2. Many of the discrepancies were thought to originate primarily from $3 \mathrm{D}$ effects such as glancing cowl shock-sidewall boundary layer interactions, spillage over the sidewall, and sidewall bleed. Therefore, a balance should be struck between expending resources on a high fidelity $2 \mathrm{D}$ simulation, and the inherent limitations of $2 \mathrm{D}$ analysis.

3. The solutions were fairly insensitive to turbulence models, grids and bleed models. Overall, the $\mathrm{k}-\varepsilon$ turbulence model, and the bleed models based on unchoked bleed hole discharge coefficients or uniform velocity are recommended.

4. 2D Navier-Stokes appears to be a useful tool for the design and analysis of supersonic inlets. It provides a higher fidelity simulation of the inlet flowfield than inviscid methods, in a reasonable turnaround time.

\section{References}

'Seddon, J. and Goldsmith, E. L., Intake Aerodynamics, AIAA Education Series, AIAA, New York, 1985.

${ }^{2}$ Goldsmith, E. L. and Seddon, J., ed., Practical Intake Aerodynamic Design, AIAA Education Series, AIAA, New York, 1993.

${ }^{3}$ Sanders, B. W. and Mitchell, G. A., "Throat Bypass Bleed Systems for Increasing the Stable Airflow Range of a Mach 2.50 Axisymmetric Inlet with 40 Percent Internal Contraction," NASA TM-X-2779, 1973.

${ }^{4}$ Bowditch, D. N., "Some Design Considerations for Supersonic Cruise Mixed Compression Inlets." NASA TM X-71460, 1973.

${ }^{5}$ Wasserbauer, J. F., Meleason, E. T. and Burstadt, P. L., "Experimental Investigation of The Performance of a Mach 2.7 Two Dimensional Bifurcated Duct Inlet with 30 Percent Internal Contraction," NASA TM to be released, 1995.

${ }^{6}$ Presley, L. L., "Internal Flow Calculations for Axisymmetric Supersonic Inlets at Angle of Attack," AIAA 75-1214, 1975.

${ }^{7}$ Vadyak, J., Hoffman, J. D., and Bishop, A. R., "Three Dimensional Flow Simulations for Supersonic Mixed-Compression Inlets at Incidence," AIAA J., Vol. 22, No. 7, 1984, pp. 873-881.
${ }^{8}$ Reyhner, T. A. and Hickcox, T. E., "Combined Viscous-Inviscid Analysis of Supersonic Inlet Flowfields," J. Aircraft, Vol. 9, No. 8, 1972, pp. 589595.

${ }^{9}$ Anderson, B. H. and Towne, C. E., "Numerical Simulation of Supersonic Inlets Using a ThreeDimensional Viscous Flow Analysis," AIAA 80-0384, 1980.

${ }^{10}$ Buggeln, R. C., McDonald, H., Kreskovsky, J. P., and Levy, R., "Computation of Three Dimensional Viscous Supersonic Flow in Inlets," AIAA 80-0194, 1980.

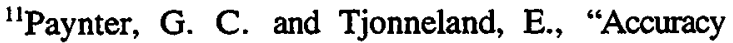
Issues in the Prediction of Supersonic Inlet Flows," ASME 92-GT-400, 1992.

${ }^{12}$ Knight, D. D., "Numerical Simulation of Realistic High-Speed Inlets Using the Navier-Stokes Equations," AIAA J., Vol. 15, No. 11, 1977, pp. 15831589.

${ }^{13}$ Chyu, W. J., Kawamura, T., and Bencze, D. P., "Calculation of External-Internal Flow Fields for Mixed-Compression Inlets," NASA TM 88362, 1986.

${ }^{14}$ Shigematsu, J., Yamamoto, K., Shiraishi, K. and Tanaka, A., "A Numerical Investigation of Supersonic Inlet Using Implicit TVD Scheme," AIAA-90-2135, 1990.

${ }^{15}$ Saunders, J. D. and Keith, T. G., Jr., "Results From Computational Analysis of a Mixed Compression Supersonic Inlet," NASA TM-104475 and AIAA-912581, 1991.

${ }^{16}$ Reddy, D. R. and Weir, L. J., "ThreeDimensional Viscous Analysis of a Mach 5 Inlet and Comparison with Experimental Data," J. Propulsion and Power, Vol. 8, No. 2, 1992, pp. 432-440.

${ }^{17}$ Fujimoto, A., Niwa, N. and Sawada, K., "Numerical Investigation of Supersonic Inlet with Realistic Bleed and Bypass Systems," J. Propulsion and Power, Vol. 8, No. 4, 1992, pp. 857-861.

${ }^{18}$ Omi, J., Shiraishi, K., Sakata, K., Murakami, A., Honami, S. and Shigematsu, J., "Two-Dimensional Numerical Simulation for Mach-3 Multishock AirIntake with Bleed Systems," AIAA-93-2306, 1993.

${ }^{19}$ Fujimoto, A. and Niwa, N., "Experimental and Numerical Investigation of Mach 2.5 Supersonic Mixed Compression Inlet," AIAA 93-0289, 1993.

${ }^{20}$ Chung, J., "Numerical Simulation of a MixedCompression Supersonic Inlet Flow," AIAA-94-0583, 1994.

${ }^{21}$ Grasso, F. and Marini, M., "Multiblock Implicit Total Variation Diminishing Solution of High-Speed Internal Flows," J. Propulsion and Power, Vol. 9, No. 2, 1993, pp. 255-262. 
${ }^{22}$ Chyu, W. J., Howe, G. W. and Shih, T. I-P., "Bleed Boundary Conditions for Numerically Simulated Mixed-Compression Supersonic Inlet Flow," $J$. Propulsion and Power, Vol. 8, No. 4, 1992, pp. 862868.

${ }^{23}$ Mayer, D. W. and Paynter, G. C., "Boundary Conditions for Unsteady Supersonic Inlet Analyses," ISABE-93-7104, 1993.

${ }^{24}$ Freskos, G. and Penanhoat, O., "Numerical Simulation of the Flow Field Around Supersonic AirIntakes," ASME 92-GT-206, 1992.

${ }^{25}$ Cooper, G. K. and Sirbaugh, J. R., "PARC Code: Theory and Usage," AEDC-TR-89-15, 1989.

${ }^{26} \mathrm{~A}$ User's Guide to NPARC, Version 2.0, NPARC Alliance, 1994.

${ }^{27}$ Dowell, E. W. Jr. and McClure, M. D., "3D INGRID: Interactive Three-Dimensional Grid Generation," AEDC-TR-87-40, 1988.

${ }^{28}$ Chien, K.-Y., "Predictions of Channel and Boundary-Layer Flows with a Low Reynolds Number Turbulence Model," AIAA J., Vol. 10, No. 1, 1982, pp. 33-38.

${ }^{29}$ Nichols, R. H., "A Two-Equation Model for Compressible Flows," AIAA-90-0494, 1990.

${ }^{30}$ Baldwin, B. S. and Barth, T. J., "A one-Equation Turbulence Transport Model for High Reynolds Number Wall-Bounded Flows," NASA TM-102847 or AIAA-910610, 1992.

${ }^{31}$ Baldwin, B. S. and Lomax, H., "Thin Layer Approximation and Algebraic Model for Separated Turbulent Flows," AIAA-78-257, 1978.

${ }^{32}$ Paynter, G., Treiber, D. and Kneeling, W., "Modeling Supersonic Inlet Bleed Roughness," AIAA92-0269, 1992.

${ }^{33}$ Syberg, J. and Hickcox, T. E., "Design of a Bleed System for a Mach 3.5 Inlet," NASA CR-2187, 1972. 
Table I. NSS and SS1 results

\begin{tabular}{|c|c|c|c|c|c|c|}
\hline \multirow[t]{2}{*}{ configuration } & \multicolumn{3}{|c|}{ NSS } & \multicolumn{3}{|c|}{ SS1 } \\
\hline & present & data & $\begin{array}{l}\text { data ( } 2 \mathrm{D} \\
\text { equivalent) }\end{array}$ & present & data & $\begin{array}{l}\text { data ( } 2 \mathrm{D} \\
\text { equivalent) }\end{array}$ \\
\hline bleed mass flow rates (\% capture) & & & & & & \\
\hline ramp shoulder & 1.43 & 1.40 & 1.40 & 1.55 & 1.40 & 1.40 \\
\hline mid diffuser & 0.48 & 1.04 & 0.55 & 1.04 & 1.26 & 0.98 \\
\hline throat & 1.35 & 2.20 & 1.61 & 2.11 & 2.70 & 1.99 \\
\hline forward sidewall & - & 1.52 & - & - & 2.06 & - \\
\hline TOTAL & 3.27 & 6.16 & 3.56 & 4.72 & 7.42 & 4.37 \\
\hline spillage (\% capture) & 1.97 & 4.6 & & 1.97 & 4.6 & \\
\hline $\begin{array}{l}\text { total pressure recovery, supersonic } \\
\text { ( } 5 \text { element throat rake) }\end{array}$ & 0.969 & & & 0.971 & & \\
\hline $\begin{array}{l}\text { cowl shock impingement } \\
\left(\mathrm{h}_{\mathrm{c}} \text { ahead of ramp shoulder }\right)\end{array}$ & 0.042 & 0.035 & & 0.042 & 0.035 & \\
\hline
\end{tabular}

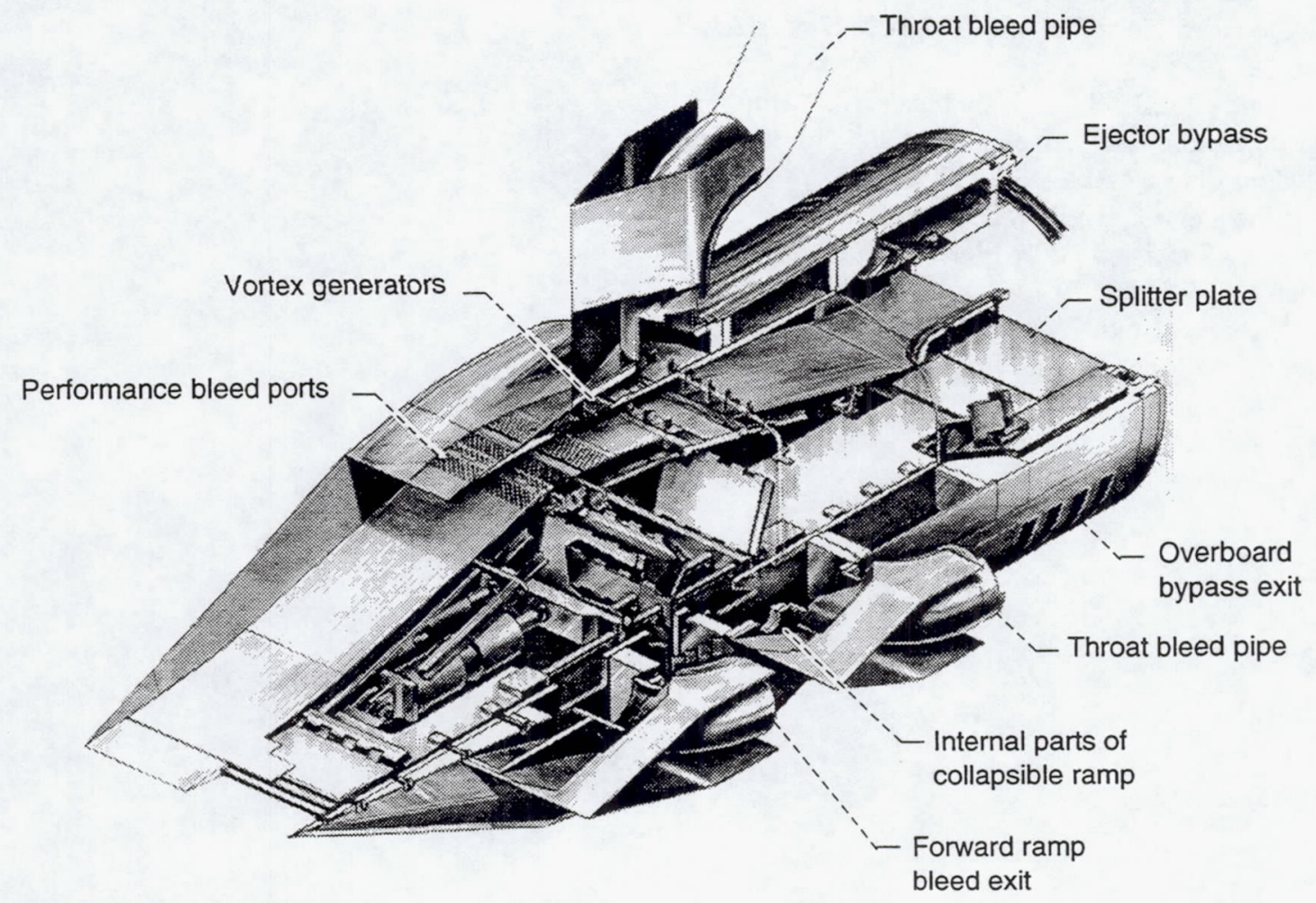

Fig. 1. Isometric cut-away view of inlet model. 


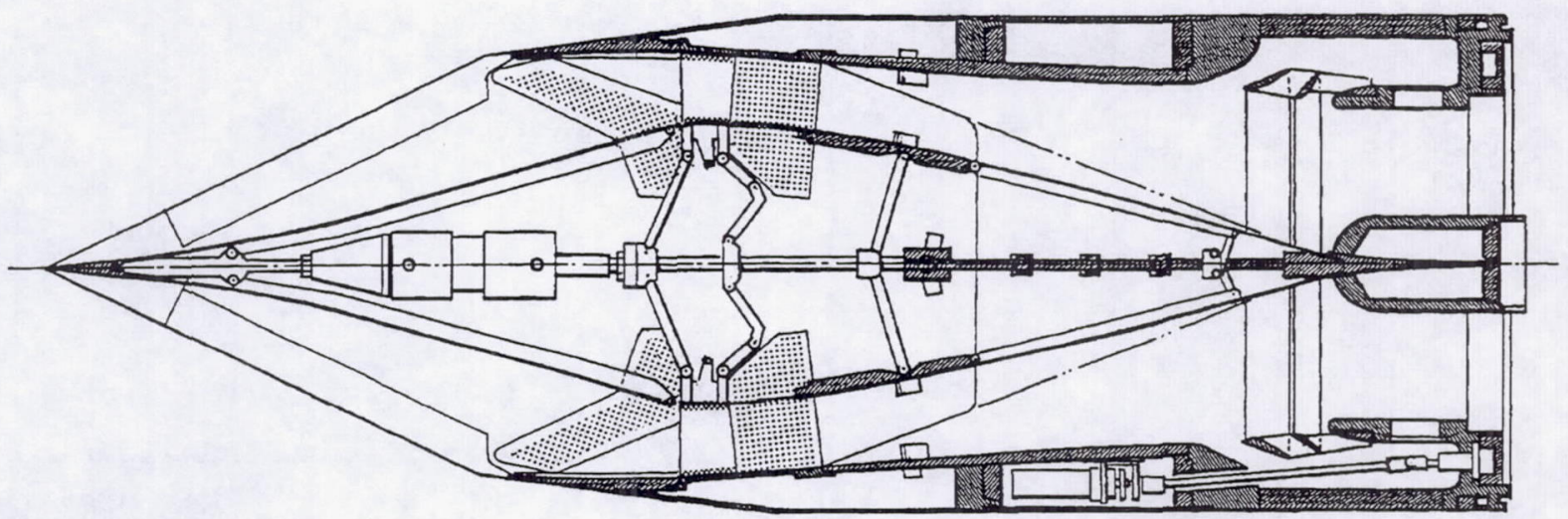

Fig. 2. Cross-sectional view of inlet model.

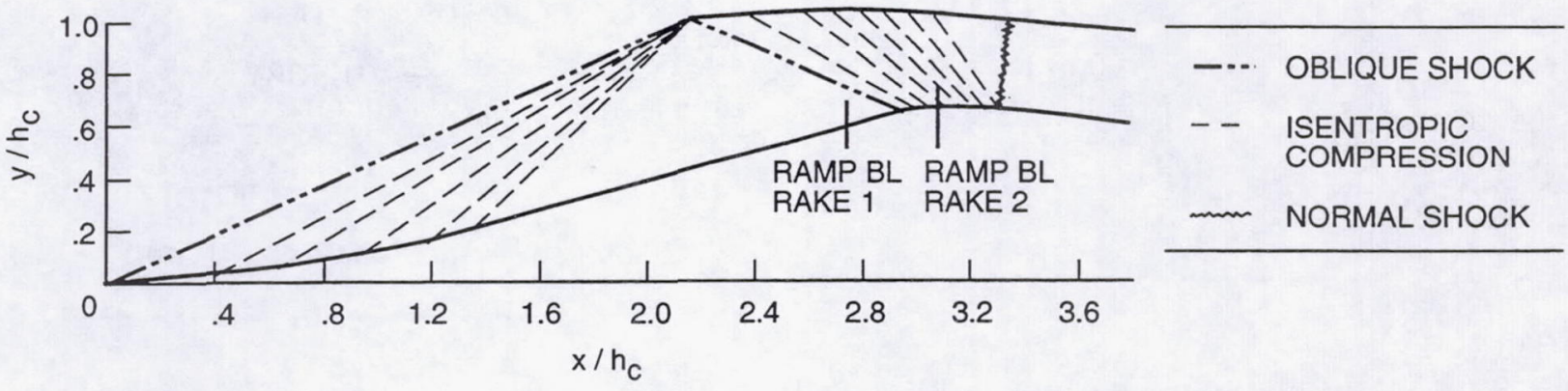

Fig. 3. Theoretical shock structure at on-design critical operation.

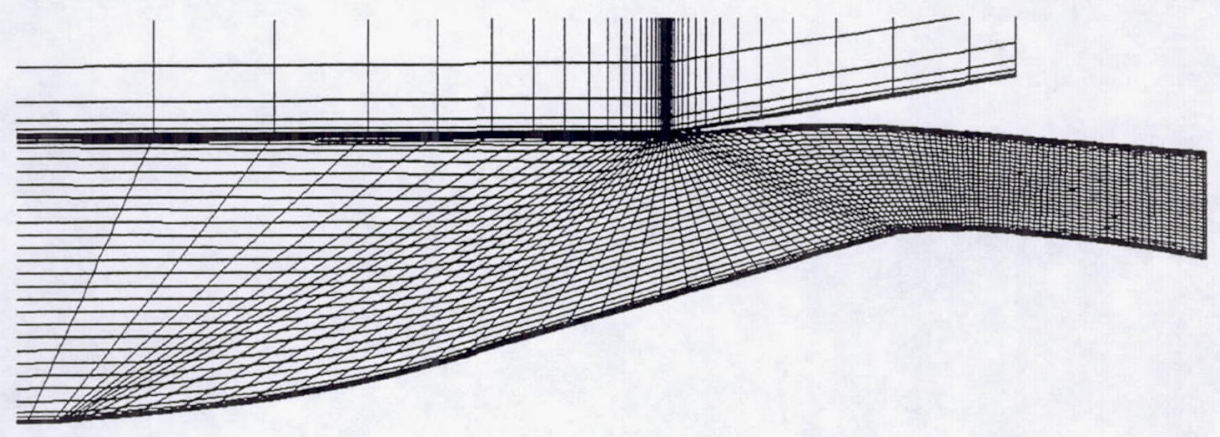

Fig. 4. Computational grid, 'improved'. Every other grid line shown for clarity. 

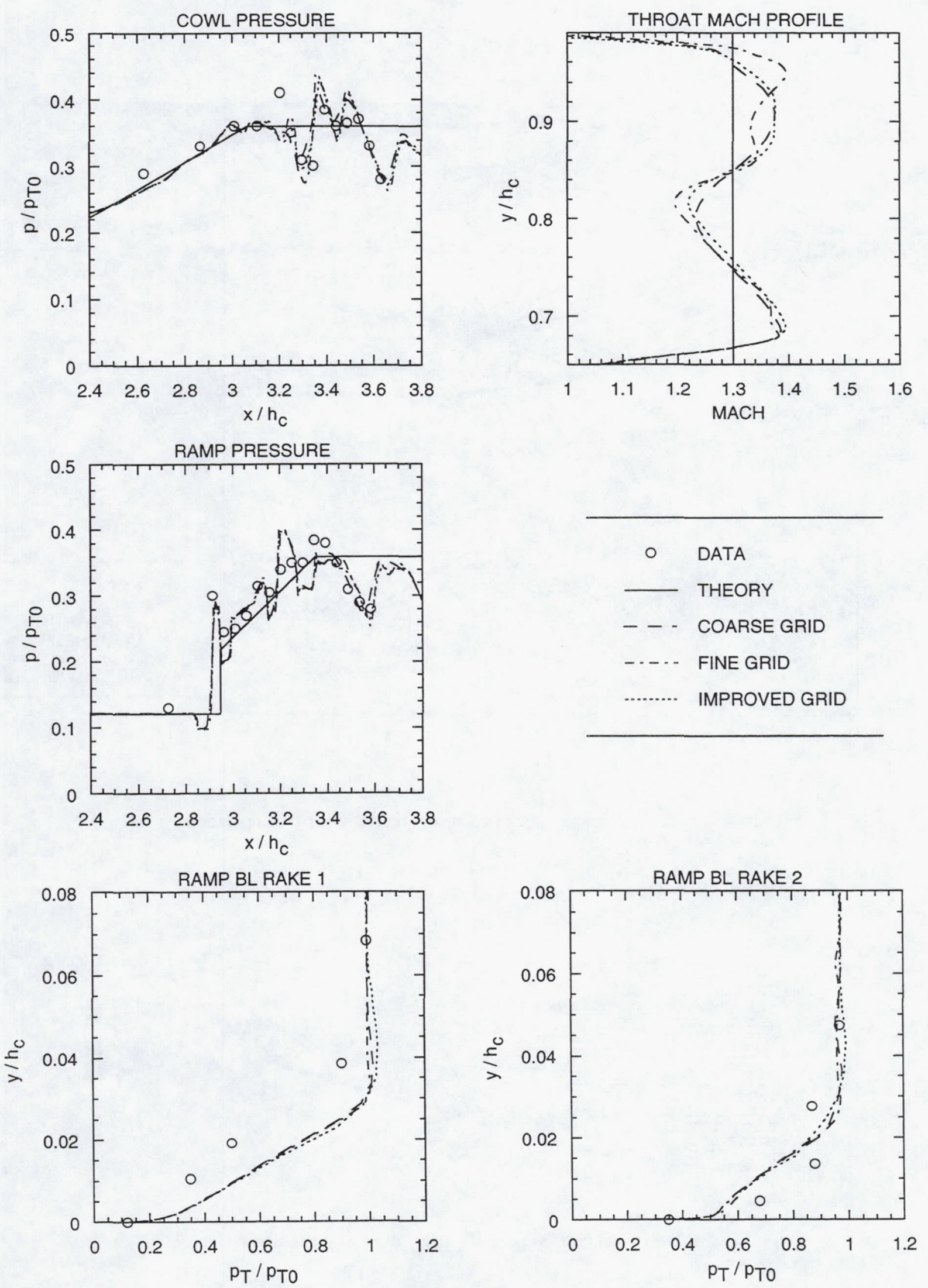

Fig. 5. Grid sensitivity, NSS configuration 

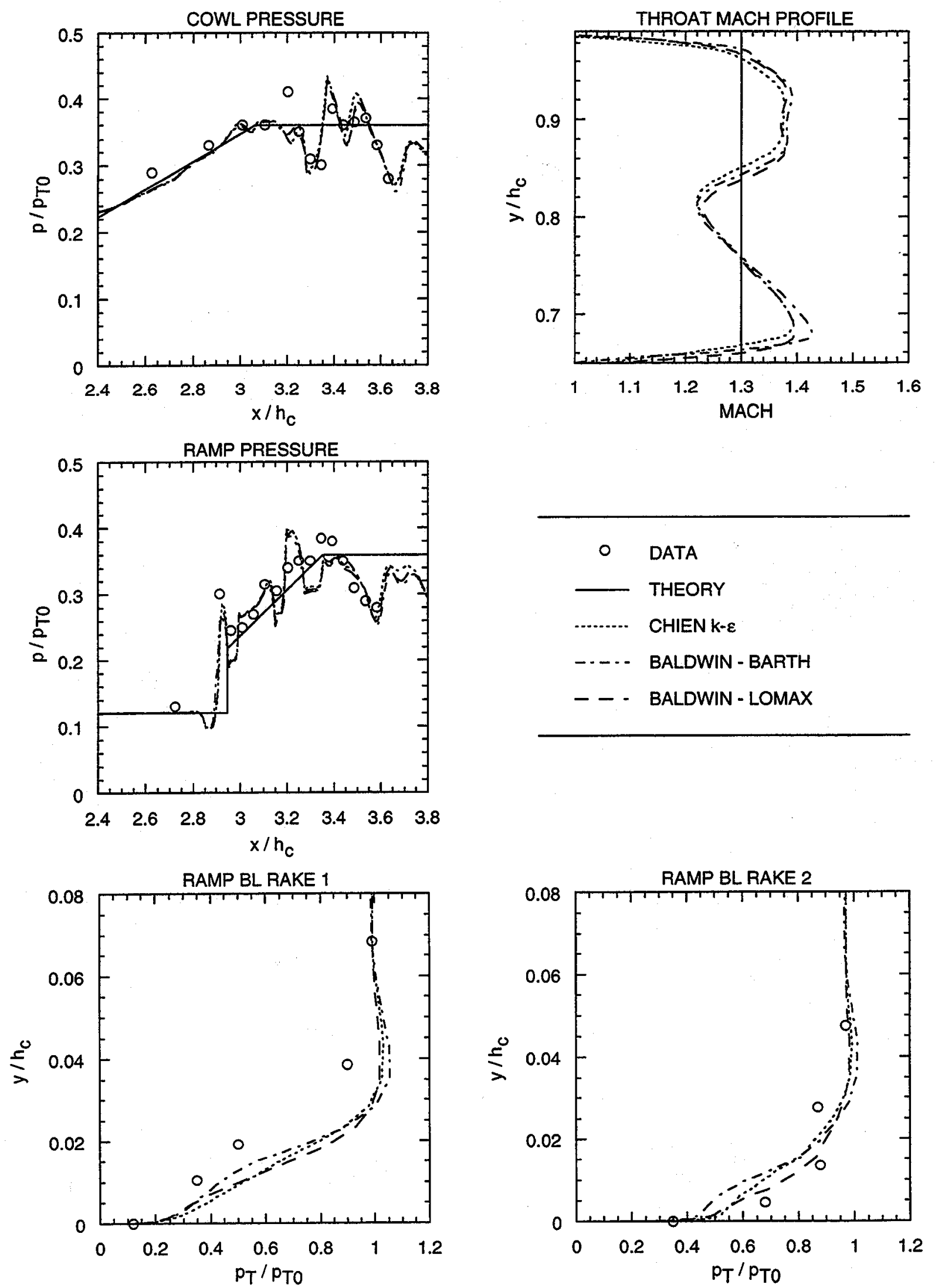

Fig. 6. Turbulence model parametrics, NSS configuration 

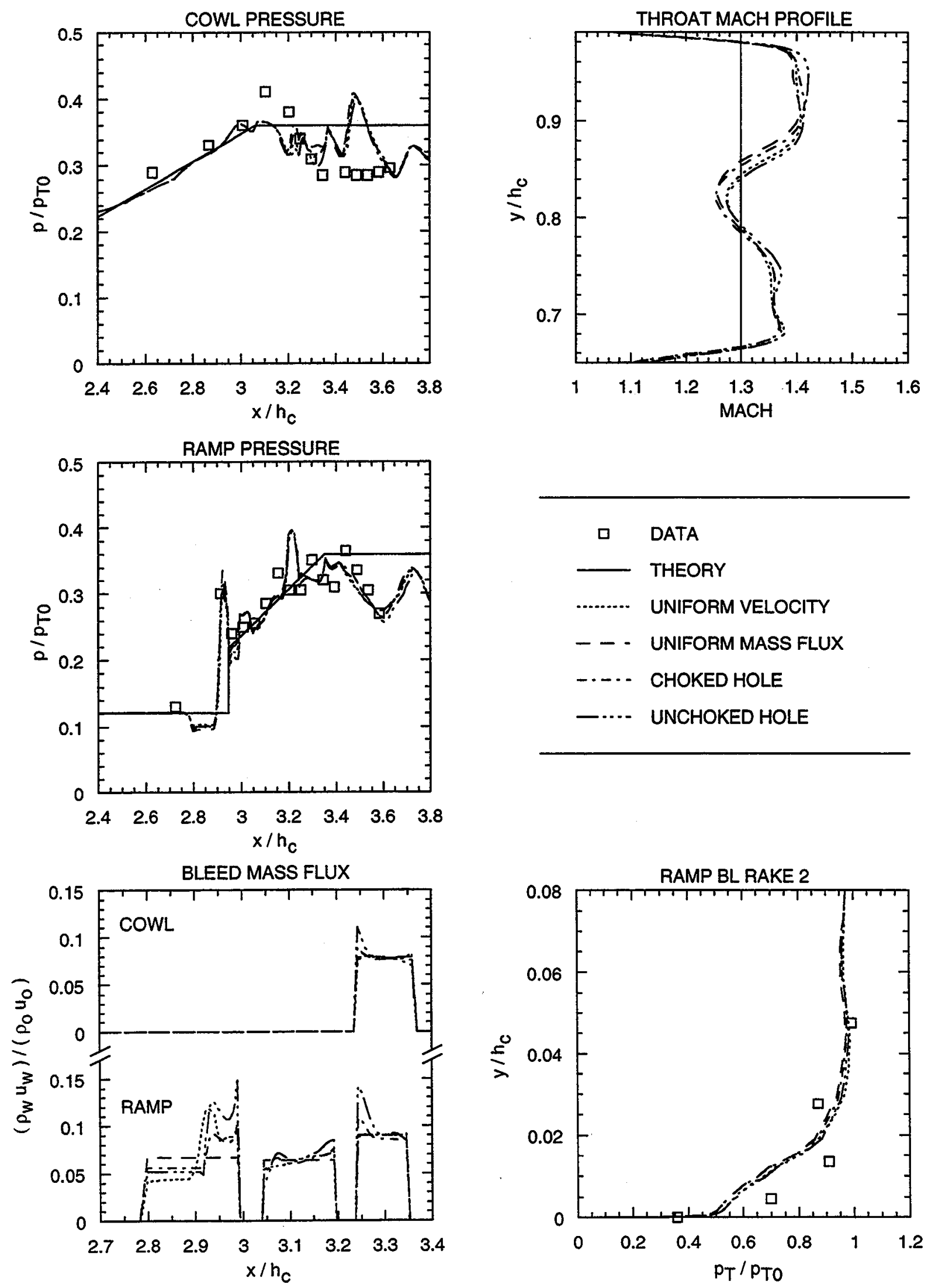

Fig. 7. Bleed model parametrics, SS1 configuration

American Institute of Aeronautics and Astronautics 
MACH CONTOURS AND BLEED MASS FLUX VECTORS
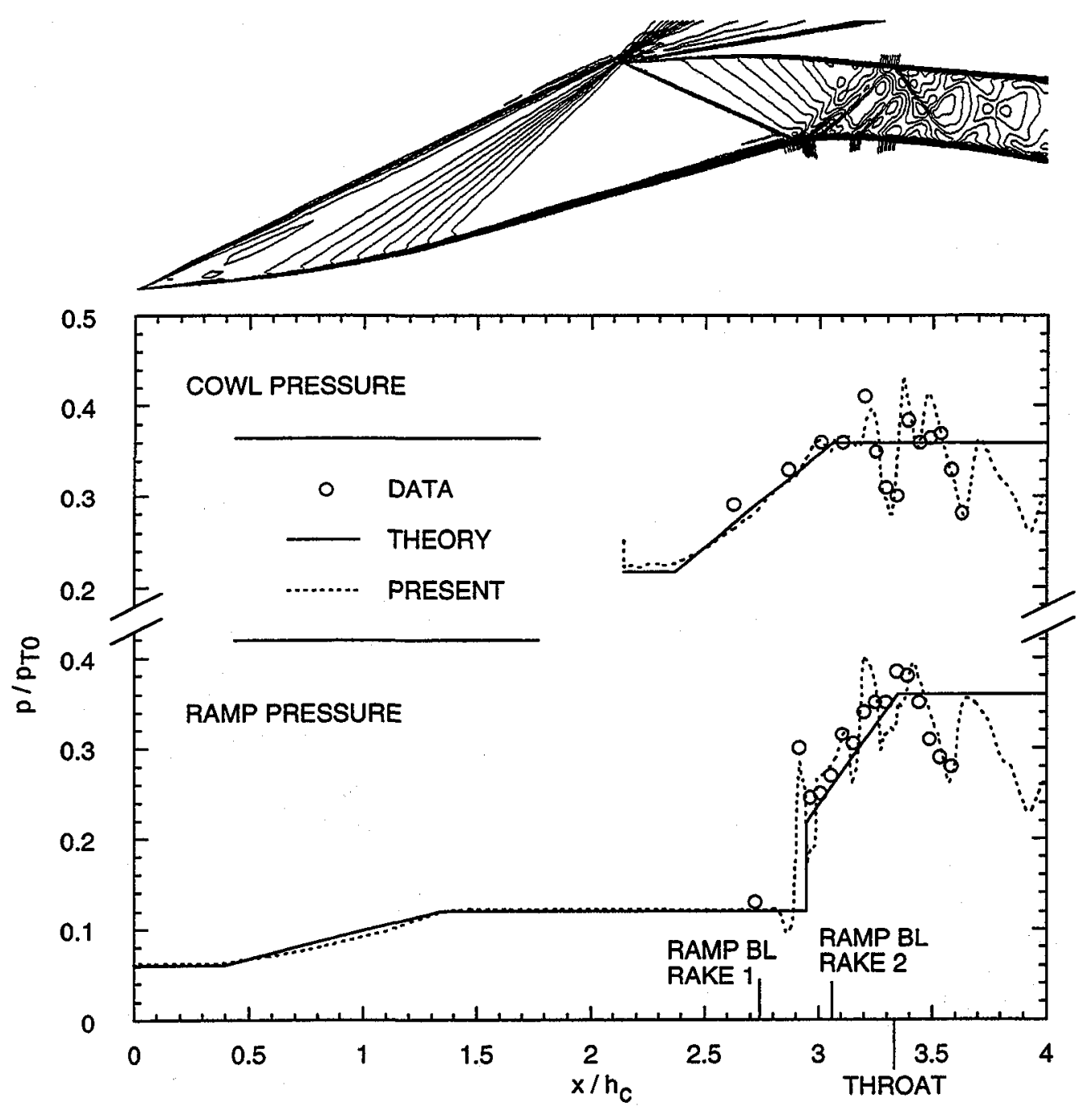

RAMP BL RAKE 1

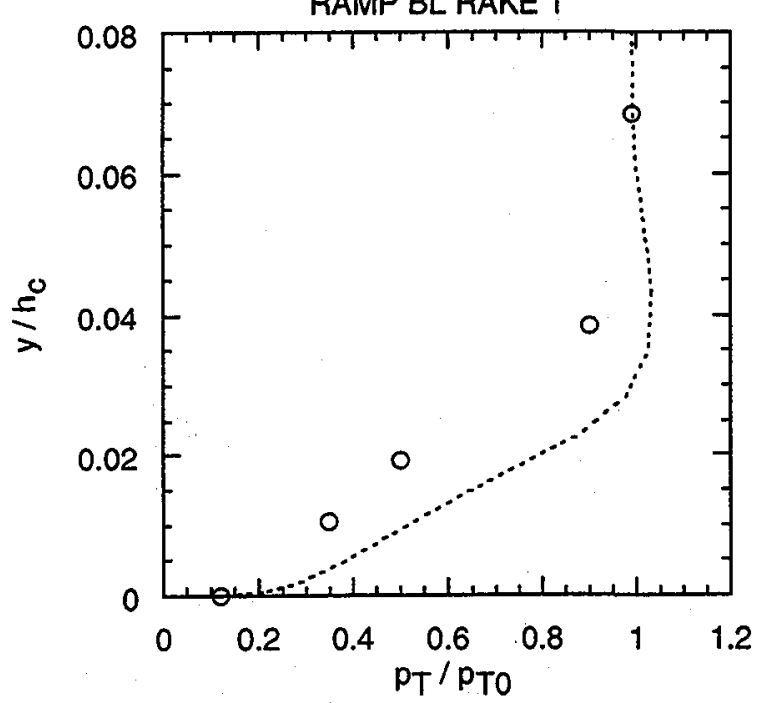

RAMP BL RAKE 2

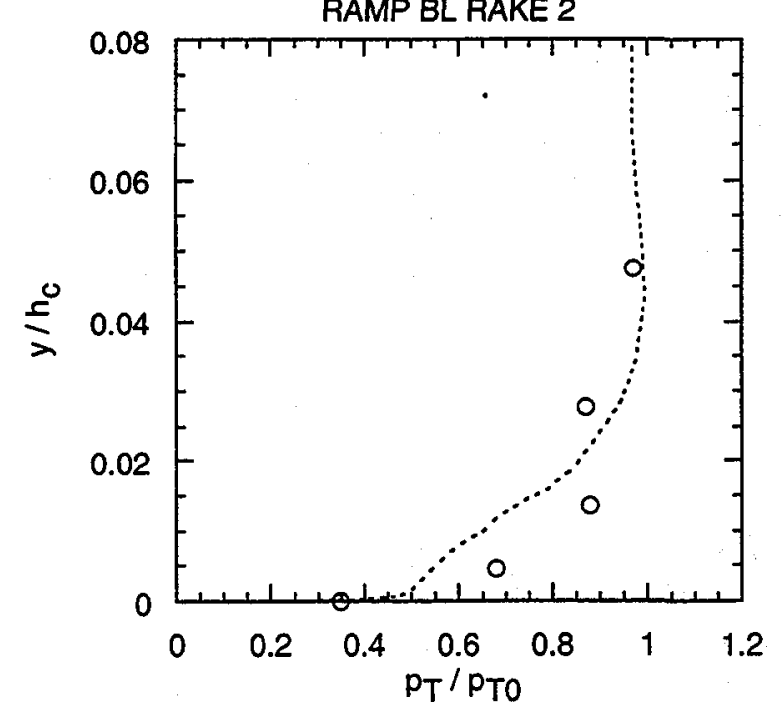

Fig. 8. NSS configuration results 


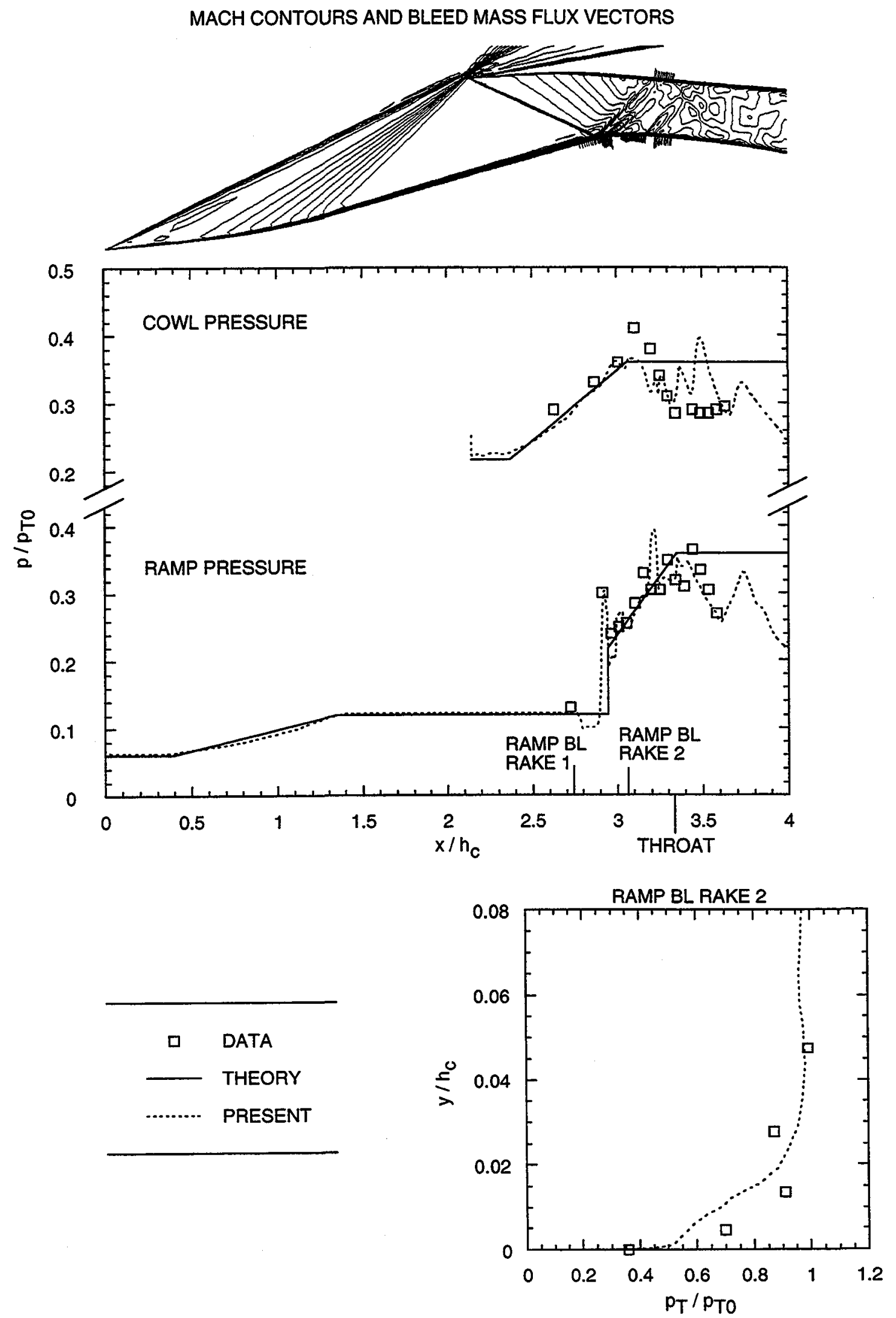

Fig. 9. SS1 configuration results 


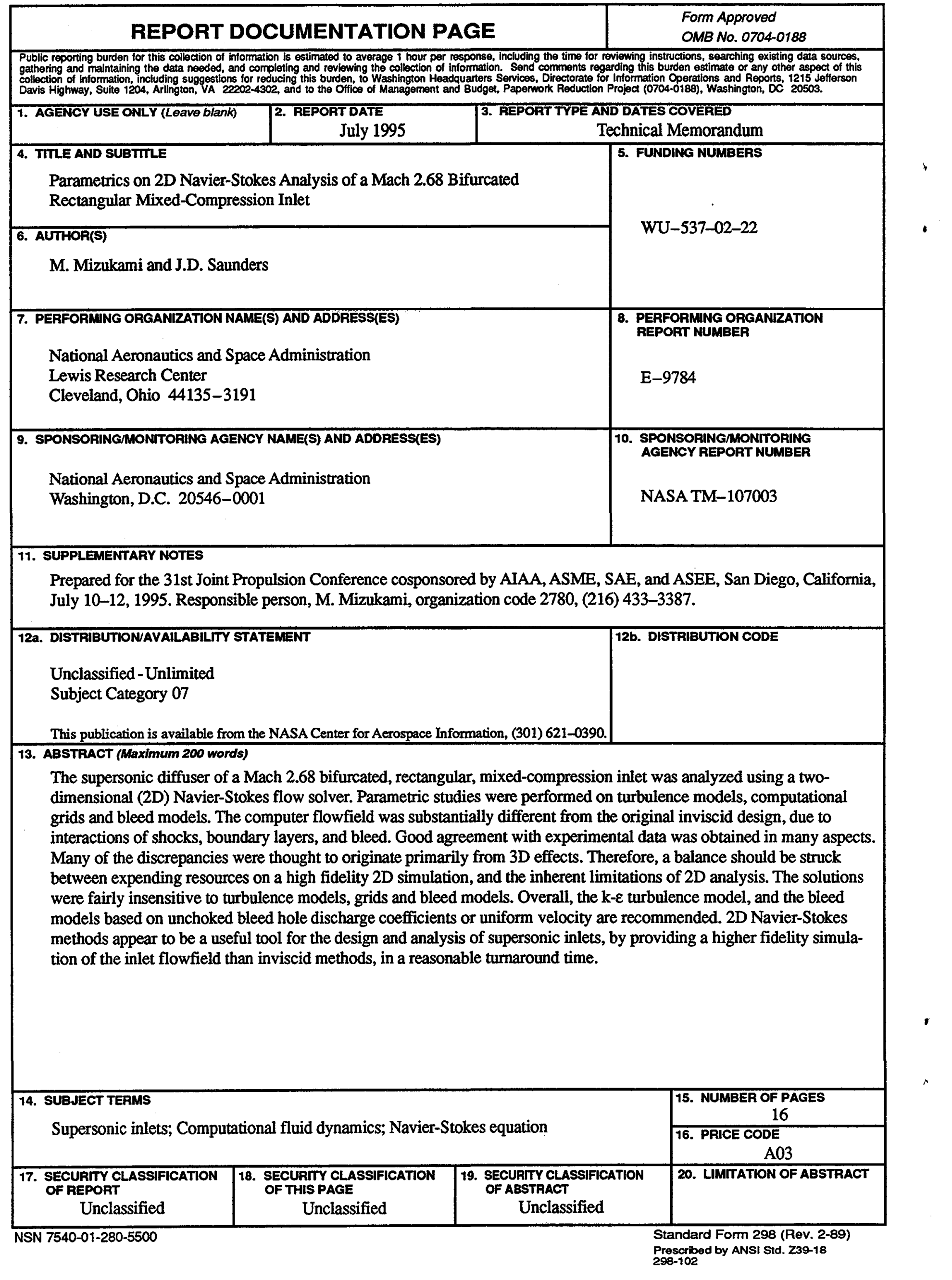


National Aeronautics and

Space Administration

Lewis Research Center

21000 Brookpark Rd.

Cleveland, $\mathrm{OH}$ 44135-3191

Official Business

Penalty for Private Use $\$ 300$

POSTMASTER: If Undeliverable - Do Not Return 